\title{
Trastornos por estrés debido a la cuarentena durante la pandemia por la COVID-19
}

Candy Palomino-Oré* 1,a; Jeff Huarcaya-Victoria $2,3,4, \mathrm{~b}$

\section{RESUMEN}

La pandemia actual por la COVID-19 presenta ciertas características que podrían incrementar los niveles de reacción al estrés tanto en la población en general como en los trabajadores de salud. Las personas que se encuentran en cuarentena son particularmente vulnerables a complicaciones neuropsiquiátricas debido al distanciamiento gradual, por lo que es más probable que aparezcan trastornos como la depresión y la ansiedad. Se sabe que, en el contexto de otros desastres, el trastorno por estrés postraumático (TEPT) puede aparecer hasta en el 30-40\% de todas las personas afectadas. El objetivo de este artículo es realizar una revisión narrativa de las consecuencias estresantes en la salud mental producidas por la cuarentena durante las epidemias y pandemias pasadas y lo que ocurre actualmente en la pandemia por la COVID-19.

Palabras clave: Infecciones por coronavirus; Pandemias; Población; Personal de salud; Cuarentena (Fuente: DeCS BIREME).

\section{Stress disorders caused by quarantine during the COVID-19 pandemic}

\section{ABSTRACT}

The current COVID-19 pandemic has certain characteristics that could increase the levels of stress reaction in both the general population and health workers. People in quarantine are particularly vulnerable to neuropsychiatric complications due to gradual distancing, making disorders such as depression and anxiety more likely to appear. It is known that, in the context of other disasters, post-traumatic stress disorder (PTSD) occurred in up to 30-40 \% of all affected people. This article aims is to carry out a narrative review of the stress consequences on mental health due to quarantine during past epidemics and pandemics, and during the current COVID-19 pandemic.

Keywords: Coronavirus infections; Pandemics; Population; Health personnel; Quarantine (Source: MeSH NLM).

1 Instituto Nacional de Salud Mental Honorio Delgado - Hideyo Noguchi, Departamento de Investigación, Docencia y Atención Especializada de Niños y Adolescentes. Lima, Perú.

2 Universidad de San Martín de Porres, Facultad de Medicina, Centro de Investigación en Salud Pública. Lima, Perú.

3 Universidad Nacional Mayor de San Marcos, Facultad de Medicina de San Fernando, Departamento Académico de Psiquiatría. Lima, Perú. 4 Hospital Nacional Guillermo Almenara Irigoyen - EsSalud, Departamento de Psiquiatría, Unidad de Psiquiatría de Enlace. Lima, Perú.

a Médico especialista en Psiquiatría de niños y adolescentes.

b Médico especialista en Psiquiatría.

* Autor corresponsal. 


\section{INTRODUCCIÓN}

La infección por el nuevo coronavirus SARS-CoV-2, también conocida como COVID-19, ha producido hasta el 13 de abril del 2020 un total de 1773084 casos confirmados y 111652 muertes en todo el mundo (1). En el Perú, hasta el 14 de abril del 2020 se han reportado 10303 casos confirmados y 230 fallecidos ${ }^{(2)}$. Estas cifras, junto con la imprevisibilidad de la pandemia y la incertidumbre de cuándo y cómo controlar la enfermedad, generan sentimientos negativos ${ }^{(3)}$. Según la experiencia de epidemias y pandemias pasadas, pueden surgir serias preocupaciones como el miedo a la muerte, sentimientos de soledad e irritabilidad, entre otras complicaciones mentales ${ }^{(3)}$.

El método crucial para romper la cadena de contagio es el distanciamiento social, es decir, la separación efectiva de las personas infectadas y de aquellos con sospecha de infección. Este proceso de separación se tiene que realizar de diferentes maneras. Mientras que el aislamiento no es absoluto, sí se deben generar medidas que limiten el contacto y exposición. El distanciamiento social también se refiere a todas las medidas que buscan reducir el contacto, como el aislamiento y la cuarentena ${ }^{(4)}$.

Las personas que se encuentran en cuarentena son particularmente vulnerables a complicaciones neuropsiquiátricas porque las personas se distancian gradualmente entre sí. En ausencia de comunicación interpersonal, es más probable que la depresión y la ansiedad ocurran y empeoren. Además, la cuarentena reduce la disponibilidad de una intervención psicosocial oportuna ${ }^{(5,6)}$. Toda la familia de desórdenes relacionados con el estrés debe ser de especial consideración: desde el trastorno por estrés agudo hasta el trastorno por estrés postraumático (TEPT), que puede presentarse hasta en el 30-40 \% de las personas afectadas, como ha ocurrido en el contexto de otros desastres ${ }^{(7,8)}$.

La evidencia también nos indica que las mujeres tienen más posibilidades de experimentar síntomas de TEPT (9). Además, un reciente metaanálisis informó que los adultos mayores tendrían hasta 2,11 veces más posibilidades de experimentar síntomas de TEPT en comparación con los adultos más jóvenes ${ }^{(10)}$. Así, en estas poblaciones de riesgo debemos buscar la reexperimentación de los sucesos traumáticos que se puede manifestar en ideas, pesadillas o flashbacks, todo ello acompañado de una evitación del estímulo con alteración de la actividad autonómica ${ }^{(11)}$.

Si bien actualmente son pocos los trabajos publicados sobre los efectos estresantes de la cuarentena en la salud mental en el contexto de la actual pandemia por el COVID-19, existen estudios realizados en epidemias y pandemias pasadas. Se ha documentado la presencia de síntomas estresantes en diversos estudios realizados en epidemias pasadas, como la del virus del Ébola (EVE) ${ }^{(12-14)}$, influenza por H1N1 (15-17) y el síndrome respiratorio agudo grave (SARS) ${ }^{(18-20)}$ (Figura 1). El objetivo de este artículo es realizar una revisión narrativa de las consecuencias estresantes en la salud mental producidas por la cuarentena en brotes infecciosos pasados y lo que ocurre actualmente en la pandemia por la COVID-19.

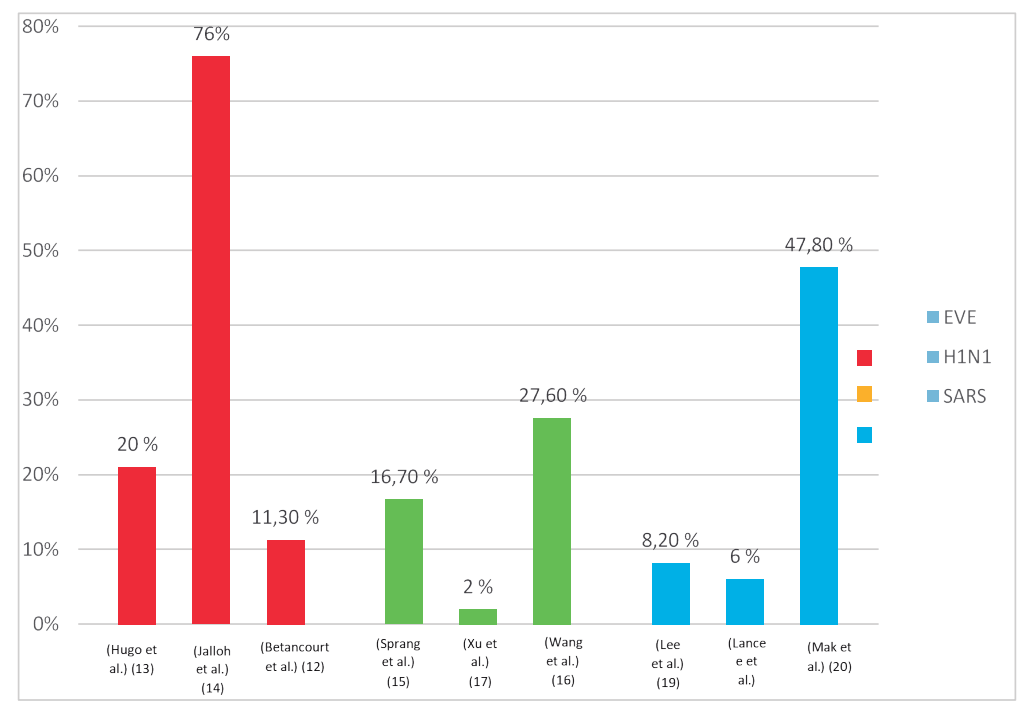

Figura 1. Prevalencias de síntomas de trastorno de estrés postraumático según diversos estudios en otras epidemias

EVE: Enfermedad por el virus del Ébola

SARS: Síndrome respiratorio agudo grave 


\section{Estrategia de búsqueda}

Se realizó una revisión narrativa a partir de una búsqueda en Pubmed de todos los artículos publicados del 01 de diciembre del 2019 hasta el 13 de abril del 2020, empleando los siguientes términos: (epidemic) AND (pandemic) AND (post traumatic stress disorder OR stress disorders OR mental health disorders). Se seleccionaron aquellos artículos que contenían información relacionada a trastornos por estrés asociados a un brote epidémico y/o pandémico escritos en inglés. Se realizó un cribado de los artículos elegibles y se revisaron los títulos y resúmenes de los artículos encontrados. De los 45 artículos encontrados, 13 se descartaron por no tener relación directa con el tema y uno por no estar en inglés o español. Finalmente, se incluyeron 31 artículos.

\section{Trastornos por estrés durante y luego de la cuarentena en epidemias y pandemias pasadas}

La implementación de una estricta cuarentena nunca antes vista en China mantuvo un largo número de personas en aislamiento y afectó muchos aspectos de sus vidas. Los resultados de diversos estudios durante epidemias pasadas sugieren que el impacto psicológico de la cuarentena es amplio, sustancial y puede durar mucho tiempo después de la exposición ${ }^{(21)}$.

Algunos brotes infecciosos previos han provocado graves consecuencias físicas y mentales en los individuos afectados (sobrevivientes, viudos, huérfanos, trabajadores de salud, entre otros), como ocurrió con la enfermedad por el virus del Ébola (EVE) que se inició en la República del Congo y fue declarado como emergencia de salud pública entre los años 2014 y 2016. De igual manera, la estigmatización, el aislamiento social, el deterioro socioeconómico y la muerte de los parientes se convirtieron en factores de riesgo relacionados con los problemas de salud mental que se presentaron posteriormente en los sobrevivientes, sus familiares y los trabajadores de la salud.

Diversos estudios demostraron que entre $27,5 \%$ al $83,3 \%$ de individuos afectados por EVE presentaron síntomas de ansiedad y entre 12 a $75 \%$ de ellos presentaron síntomas depresivos. En estas investigaciones se observó que tanto los síntomas de depresión como los de TEPT podrían alterar el juicio, por lo que constituían un riesgo para contraer la EVE debido a que las actividades preventivas, en esta población, se acataban en menor medida. Se observó también que los altos niveles de ansiedad se asociaron con más actividades de prevención, como el lavado de manos y la búsqueda de información confiable, lo que sugirió que las personas ansiosas podían mantener altos niveles de vigilancia o preocupación sobre la EVE y, de esta manera, disminuir las tasas de contagio ${ }^{(22) .}$
Por otro lado, durante el brote del síndrome respiratorio agudo grave (SARS) los sobrevivientes fueron los más afectados: la experiencia de ser testigos de eventos adversos durante la hospitalización, la incertidumbre del pronóstico y la necesidad de atención en la Unidad de Cuidados Intensivos (UCl) constituyeron situaciones terroríficas para ellos (20). Un estudio de seguimiento hecho en Hong Kong comparó el nivel de estrés reportado durante el brote del SARS y un año después en los sobrevivientes; se evidenció que estos niveles permanecieron elevados en vez de haberse mitigado por el tiempo transcurrido. Además, el $90 \%$ del personal de salud sobreviviente puntuó por encima del umbral en el General Health Questionnaire (GHQ-12) ${ }^{(19)}$.

En un metaanálisis de 22 estudios hechos en diez países que incluyó personas con SARS, enfermedad del Ébola, influenza H1N1 (2009 al 2010), síndrome respiratorio de medio este (MERS) e influenza equina, se encontró que la cuarentena en el personal de salud que tuvo contacto con SARS fue el factor más predictivo para la aparición de síntomas de trastorno de estrés agudo. Además, se reportaron varias respuestas negativas durante el periodo de cuarentena: temor $(20 \%)$ nerviosismo (18\%), tristeza (18\%) y culpa (10\%). Asimismo, durante el brote de influenza equina, aproximadamente $34 \%$ de los dueños de caballos que estuvieron en cuarentena por varias semanas, reportaron un alto nivel de estrés psicológico comparado con alrededor de $12 \%$ de la población australiana en general (21).

En un estudio en el que participaron 1082 estudiantes universitarios durante la pandemia de H1N1, se encontraron como predictores significativos de síntomas de estrés como ser mujer, estar infectado con influenza H1N1 y tener familia, amigos o conocidos con la enfermedad. Debido a que este brote tenía como población de riesgo a los jóvenes, muchos estudiantes experimentaron una variedad de reacciones de estrés en el brote de influenza H1N1 y alrededor de $2 \%$ de los estudiantes desarrollaron TEPT ${ }^{(17)}$.

Lee et al. ${ }^{(23)}$ publicaron recientemente un estudio con el objetivo de evaluar el estrés inmediato y el impacto psicológico experimentado por los trabajadores de salud sometidos a cuarentena durante el brote del MERS. Evaluaron a 1800 médicos que obtuvieron puntajes elevados de estrés y problemas en el sueño que se agravaron en el hogar después de la cuarentena. Los investigadores resaltan que se necesita una intervención siquiátrica rápida y continua durante los brotes de enfermedades infecciosas de alta mortalidad.

Trastornos por estrés en la pandemia por la COVID-19

La pandemia actual por la COVID-19 tiene algunas características que pueden favorecer a la aparición de mayores niveles de reacción al estrés comparado 
con otras pandemias: es un virus nuevo, lo que genera cierta incertidumbre sobre su manejo y lleva a un futuro impredecible. El número de casos positivos y de fallecimientos crece día a día y la información se reproduce fácilmente en las redes sociales. Actualmente, a través de internet y de los teléfonos inteligente existe mayor información, sin embargo, ese mecanismo también facilita la desinformación y la transmisión de mitos (24). En este contexto, no nos debe extrañar que en los estudios que se realicen se encontrarán altos niveles de reacción al estrés.

Un estudio evaluó el estado de salud mental de 398 padres de niños que experimentaron la contención de la enfermedad mediante el aislamiento o la cuarentena y encontraron que el $30 \%$ y el $25 \%$ de los padres reunieron criterios para TEPT ${ }^{(25)}$.

En otra investigación con 2091 participantes, 96 (4,6 \%) reportaron gran número de síntomas de TEPT y en personal de salud se reportó $4,4 \%$. Los factores de riesgo que se identificaron fueron el sexo femenino, tener historia de exposición en Wuhan, estar en los grupos de alto riesgo de infección y tener pobre calidad de sueño. Las personas que ahora residen en la provincia de Hubei, el área con mayor cantidad de afectados, reportaron los más altos niveles de TEPT. Este resultado es consistente con un estudio sobre el impacto psicológico del SARS, que demostró que los residentes en áreas con altas prevalencias de SARS eran más susceptibles a desarrollar un TEPT (26).

A finales de enero del 2020, un estudio realizado en China encuestó a un total de 52730 personas, y el $35 \%$ de participantes reportó una reacción al estrés. Las variables que más se asociaron a los mayores niveles de estrés fueron ser mujer y pertenecer a los grupos etarios de entre 18 y 30 años, y los mayores de 60 años. Las puntuaciones más altas entre el grupo de adultos jóvenes podrían explicarse porque ellos obtienen una gran cantidad de información de las redes sociales, lo cual puede desencadenar fácilmente el estrés. De otro lado, dado que la tasa de mortalidad más alta por la COVID-19 se produce en los adultos mayores, no es sorprendente que ellos tengan más posibilidades de sufrir un impacto psicológico negativo ${ }^{(27)}$. A esto debemos sumar que los adultos mayores tienen un acceso limitado a los servicios de internet y smartphones, por lo que una proporción muy pequeña de ellos se benefician de los servicios de apoyo sicosocial en línea ${ }^{(28)}$.

En otro estudio realizado por Wang et al. (29) se empleó el Impact of Event Scale-Revised (IES-R) en una muestra de 1210 personas durante la fase inicial de la pandemia en China. Los investigadores reportaron que un $24,5 \%$ presentaron un estrés psicológico mínimo; $21,7 \%$ moderado; y un $53,8 \%$, moderadosevero. El ser mujer, estudiante y tener algunos síntomas físicos se asociaron con mayores niveles de estrés ${ }^{(29)}$.
Finalmente, Liu et al. ${ }^{(30)}$ encontraron una prevalencia de TEPT de $7 \%$ en las zonas de China más afectadas por la COVID-19, valor que es mayor a lo reportado en otras epidemias, esto sugiere que la COVID-19 tuvo un impacto estresante más alto en la población general. Lo más importante de este estudio son las diferencias encontradas entre las características del TEPT según el género: las mujeres tuvieron mayor número de síntomas de hiperactividad, de recuerdos angustiosos recurrentes y de alteraciones negativas cognitivas y del estado de ánimo. Todos estos estudios remarcan la importancia que debemos prestar a la salud mental de las mujeres durante el desarrollo de la pandemia por la COVID-19.

Si la cuarentena es esencial, se deben tomar medidas para asegurar que esta experiencia sea lo más tolerable y menos estresante para las personas. Esto puede lograrse de diferentes formas:

1) Informar a las personas sobre lo que está sucediendo y por qué, y explicar cuanto tiempo continuará.

2) Brindar herramientas para realizar durante la cuarentena.

3) Asegurar que los suplementos básicos estén disponibles y reforzar el sentido de altruismo que las personas deberían sentir ${ }^{(21)}$.

Todo esto sugiere que los servicios psicológicos son importantes en la fase de rehabilitación y no deben ser olvidados mientras se espera un nuevo brote de algún virus ${ }^{(19)}$.

Necesidad de resiliencia en el personal de salud y población general ¿cómo alcanzarla?

El estrés laboral frecuentemente asociado al dolor humano y al ambiente de trabajo durante la pandemia puede impactar en el bienestar físico y mental de los trabajadores de la salud (TS) y dar como resultado una sobrecarga laboral y, en algunos casos, síntomas parecidos al TEPT. Por lo tanto, es importante que se adopten medidas preventivas, desarrollando y fomentando personas y ambientes resilientes como manera de reducir lo negativo e incrementar lo positivo en el área de la salud. La definición de resiliencia ha sido adaptada de la literatura del desarrollo psicológico y se define como la habilidad para mantener el bienestar personal y profesional a pesar del estrés laboral continuo y la adversidad ${ }^{(31)}$.

Se ha visto que nuevos episodios de trastornos siquiátricos pueden ser más prevalentes en el personal de salud con historia previa de enfermedades mentales. Estos hallazgos sugieren que incluso en el personal de salud con mayor riesgo de sufrir un trastorno mental, los esfuerzos del hospital para ofrecer un soporte práctico, emocional y proveer entrenamiento en nuevas tareas y protección 
personal mejoran significativamente la resiliencia de los trabajadores ${ }^{(18)}$.

Trabajos recientes se han enfocado en incrementar la resiliencia de los TS, con énfasis en la educación de estos para identificar sus roles, sus estresores, posibles reacciones y síntomas y/o desarrollar estrategias cognitivas y conductuales. En el modelo Anticipar, Planificar y Disuadir (APD), el riesgo de respuesta y modelo de resiliencia fue desarrollado para proveer un nuevo método basado en evidencia y, de esta manera, entender y manejar el impacto psicológico entre los TS, que incluyen estrategias para manejar todo tipo de riesgos y mejorar la resiliencia en el receptor y sus familias y que se enfocan en la resiliencia de los TS durante las fases de preincidencia, respuesta y recuperación de emergencias en salud pública; además, se propone realizar un autotriaje mediante el test PsySTART-R que rastrea la exposición continua a estresores y provee una retroalimentación confidencial, animando al individuo a usar su plan de resiliencia personal desarrollado como parte del entrenamiento APD y a buscar ayuda profesional, si lo necesitara. Este modelo se utilizó durante el brote de virus del Ébola en 2014-2015 y se demostró su viabilidad al proteger a los TS con alto riesgo de consecuencias sicológicas negativas de trauma agudo potencial y estresores cumulativos a futuro ${ }^{(32)}$.

También es importante promover la capacidad de resiliencia en la población general durante la pandemia por la COVID-19. Se ha observado que la exposición a conflictos de masa y desplazamientos mejora la capacidad de resiliencia, como sucedió en Sierra Leona, donde la población, antes expuesta a la guerra civil, mejoró su capacidad de resiliencia entre los pobladores durante el brote de EVE, y se encontraron síntomas clínicos de ansiedad y depresión "muy por debajo de lo esperado" dada la magnitud y duración de la epidemia ${ }^{(14)}$.

En comparación con la crisis ocasionada por la guerra, muchos temas estuvieron relacionados únicamente con el impacto de la EVE en las comunidades:

1) La EVE se asoció a la presencia de un estigma que no fue observado durante la guerra. que se derivó de los rumores o de la desinformación sobre la forma de trasmisión de la enfermedad y las personas que eran susceptibles a contraerla. Tanto la desinformación como el estigma no estuvieron limitados a un grupo demográfico específico ya que no se vieron diferencias entre los grupos según niveles de educación.

2) La EVE tuvo un impacto significativo en las normas culturales y expectativas, si se compara con la guerra. Las comunidades se vieron obligadas a cambiar las prácticas de inhumación para evitar el contacto con los cadáveres de los fallecidos por la infección ya que la EVE podía trasmitirse por esta vía. Para muchos, estos cambios de hábito ocasionaron un trauma psicológico al no poder despedirse de sus seres queridos y conservar sus tradiciones.
La resiliencia de la comunidad es crucial para manejar grandes conmociones como la epidemia de la EVE y facilitar la recuperación de las comunidades a corto plazo. Sin embargo, dentro del ámbito de la salud mental los esfuerzos para reforzar dicha resiliencia no atraen las mismas inversiones que en otras áreas ${ }^{(33)}$. Además, las medidas de contención que limitan la movilización de los individuos minimizan los riesgos, pero también pueden disminuir la resiliencia porque deterioran la funcionalidad del sistema por un tiempo prolongado. Por ello, el campo emergente de la evaluación y manejo de la resiliencia puede evaluar alternativas para diseñar políticas que mejoren la capacidad del sistema para enfrentarse a estos eventos, absorber el estrés, recuperarse y predecir el manejo de futuros estresores.

Se ha observado que las medidas de contención que limitan la movilización de los individuos ayudan a disminuir los riesgos, pero pueden deteriorar la funcionalidad del sistema por un tiempo prolongado y, en consecuencia, disminuir la resiliencia en la población. El campo emergente de la evaluación y manejo de la resiliencia puede evaluar alternativas para diseñar políticas que mejoren la capacidad del sistema para enfrentarse a estos eventos, absorber el estrés, recuperarse y predecir el manejo de futuros estresores.

El campo emergente de la evaluación y manejo de la resiliencia puede evaluar alternativas para diseñar políticas que mejoren la capacidad del sistema para enfrentarse a estos eventos, absorber el estrés, recuperarse y predecir el manejo de futuros estresores. Entonces, en el proceso de la contención de la COVID-19 en el Perú debemos tener especial consideración en la resiliencia de la población general y de nuestros trabajadores de salud.

\section{CONCLUSIONES}

La pandemia actual de la COVID-19 tiene algunas características que favorecen una reacción mayor al estrés si se compara con otras pandemias. En la revisión de la evidencia publicada hasta el momento encontramos que los grupos más vulnerables para desarrollar problemas de estrés son las mujeres, los adultos mayores y el personal de salud. En el proceso de la contención de esta pandemia debemos tener especial consideración en la resiliencia de la población general y de nuestro personal de salud, por lo que es prioridad desarrollar estrategias y modelos de intervención en salud mental que ayuden a disminuir el impacto psicosocial en el futuro. Es necesaria una intervención siquiátrica rápida y continua durante estados de cuarentena o inmovilización a causa de enfermedades infecciosas de alta mortalidad.

Contribución de los autores: Ambos autores participaron de la elaboración, redacción y aprobación del manuscrito.

Fuentes de financiamiento: El artículo ha sido financiado por los autores. 
Conflicto de intereses: Los autores declaran no tener ningún conflicto de interés.

\section{REFERENCIAS BIBLIOGRÁFICAS}

1. World Health Organization. Coronavirus disease 2019 (Covid-19) Situation Report - 84 [Internet]. 2020. Disponible en: https:// www. who.int/docs/default-source/coronaviruse/situationreports/20200413-sitrep-84-covid-19.pdf?sfvrsn=44f511ab_2.

2. Ministerio de Salud. Sala Situacional Covid-19 en Perú [Internet]. 2020. Disponible en: https://covid19.minsa.gob.pe/sala_situacional.asp.

3. Zandifar A, Badrfam R. Iranian mental health during the COVID-19 epidemic. Asian J Psychiatr. 2020; 51: 101990.

4. Huremovic D. Social distancing, quarantine, and isolation. In: Huremovic D, editor. Psychiatry of pandemics a mental health response to infection outbreak. Switzerland: Springer; 2019. p. 85-94.

5. Xiao C. A novel approach of consultation on 2019 Novel Coronavirus (Covid-19)-Related psychological and mental problems: structured letter therapy. Psychiatry Investig. 2020; 17(2): 175-6.

6. Huremovic D. Mental health of quarantine and isolation. In: Huremovic $D$, editor. Psychiatry of pandemics a mental health response to infection outbreak. Switzerland: Springer; 2019. p. 95-118.

7. Galea S, Nandi A, Vlahov D. The epidemiology of post-traumatic stress disorder after disasters. Epidemiol Rev. 2005; 27: 78-91.

8. Neria Y, Nandi A, Galea S. Post-traumatic stress disorder following disasters: a systematic review. Psychol Med. 2008; 38(4): 467-80.

9. Bell SA, Folkerth LA. Women's mental health and intimate partner violence following natural disaster: a scoping review. Prehosp Disaster Med. 2016; 31(6): 648-57.

10. Parker G, Lie D, Siskind DJ, Martin-Khan M, Raphael B, Crompton $D$, et al. Mental health implications for older adults after natural disasters - a systematic review and meta-analysis. Int Psychogeriatr. 2016; 28(1): 11-20.

11. León-Amenero D, Huarcaya-Victoria J. Salud mental en situaciones de desastres. Horiz Med. 2019; 19(1): 73-80.

12. Betancourt TS, Brennan RT, Vinck P, VanderWeele TJ, Spencer-Walters $\mathrm{D}$, Jeong $\mathrm{J}$, et al. Associations between mental health and ebolarelated health behaviors: a regionally representative cross-sectional survey in post-conflict Sierra Leone. PLoS One. 2016; 13(8): e1002073.

13. Hugo M, Declerck H, Fitzpatrick G, Severy N, Gbabai O, Decroo T. Posttraumatic stress reactions in Ebola virus disease survivors in Sierra Leone. Emerg Med. 2015; 5(6): 1-4.

14. Jalloh MF, Li W, Bunnell RE, Ethier KA, O'Leary A, Hageman KM, et al. Impact of Ebola experiences and risk perceptions on mental health in Sierra Leone, July 2015. BMJ. 2018; 3(2): e000471.

15. Sprang G, Silman M. Post-traumatic stress disorder in parents and youth after health-related disasters. Disaster Med Public Health Prep. 2013; 7(1): 105-10.

16. Wang $Y, X u B$, Zhao $G$, Cao $R$, He $X$, Fu $S$. Is quarantine related to immediate negative psychological consequences during the $2009 \mathrm{H} 1 \mathrm{~N} 1$ epidemic?. Gen Hosp Psychiatry. 2011; 33(1): 75-7.

17. Xu J, Zheng Y, Wang M, Zhao J, Zhan Q, Fu M, et al. Predictors of symptoms of posttraumatic stress in Chinese university students during the 2009 H1N1 influenza pandemic. Med Sci Monit. 2011; 17(7): PH60.

18. Lancee WJ, Maunder RG, Goldbloom DS. Prevalence of psychiatric disorders among Toronto hospital workers one to two years after the SARS outbreak. Psychiatric Serv. 2008; 59(1): 91-5.

19. Lee AM, Wong JG, MCAlonan GM, Cheung V, Cheung C, Sham PC, et al. Stress and psychological distress among SARS survivors 1 year after the outbreak. Can J Psychiatry. 2007; 52(4): 233-40.

20. Mak IWC, Chu CM, Pan PC, Yiu MGC, Chan VL. Long-term psychiatric morbidities among SARS survivors. Gen Hosp Psychiatry. 2009; 31(4): 318-26.
21. Brooks SK, Webster RK, Smith LE, Woodland L, Wessely S, Greenberg $\mathrm{N}$, et al. The psychological impact of quarantine and how to reduce it: rapid review of the evidence. The Lancet. 2020.

22. Cénat JM, Mukunzi JN, Noorishad P-G, Rousseau C, Derivois D, Bukaka J. A systematic review of MHPSS programs among populations affected by the Ebola virus disease. J Psychosom Res. 2020: 109966.

23. Lee SM, Kang WS, Cho AR, Kim T, Park JK. Psychological impact of the 2015 MERS outbreak on hospital workers and quarantined hemodialysis patients. Compr Psychiatry. 2018; 87: 123-7.

24. Bao Y, Sun Y, Meng S, Shi J, Lu L. 2019-nCoV epidemic: address mental health care to empower society. The Lancet. 2020; 395(10224): e37-8.

25. Hossain MM, Sultana A, Purohit N. Mental health outcomes of quarantine and isolation for infection prevention: a systematic umbrella review of the global evidence; 2020.

26. Sun L, Sun Z, Wu L, Zhu Z, Zhang F, Shang Z, et al. Prevalence and risk factors of acute posttraumatic stress symptoms during the Covid-19 outbreak in Wuhan, China. medRxiv. 2020.

27. Qiu J, Shen B, Zhao M, Wang Z, Xie B, Xu Y. A nationwide survey of psychological distress among Chinese people in the Covid-19 epidemic: implications and policy recommendations. Gen Psychiatr. 2020; 33(2): e100213.

28. Yang Y, Li W, Zhang Q, Zhang L, Cheung T, Xiang YT. Mental health services for older adults in China during the COVID-19 outbreak. Lancet Psychiatry. 2020; 7(4): e19.

29. Wang C, Pan R, Wan X, Tan Y, Xu L, Ho CS, et al. Immediate psychological responses and associated factors during the initial stage of the 2019 Coronavirus Disease (Covid-19) epidemic among the general population in China. Int J Environ Res Public Health. 2020; 17(5).

30. Liu N, Zhang F, Wei C, Jia Y, Shang Z, Sun L, et al. Prevalence and predictors of PTSS during Covid-19 outbreak in China hardest-hit areas: gender differences matter. Psychiatry Res. 2020; 287: 112921.

31. McCann CM, Beddoe E, McCormick K, Huggard P, Kedge S, Adamson $C$, et al. Resilience in the health professions: a review of recent literature. Int J Wellbeing. 2013; 3(1).

32. Schreiber M, Cates DS, Formanski S, King M. Maximizing the resilience of healthcare workers in multi-hazard events: lessons from the 20142015 Ebola response in Africa. Mil Med. 2019; 184(Suppl. 1): 114-20.

33. Alonge $\mathrm{O}$, Sonkarlay S, Gwaikolo W, Fahim C, Cooper J, Peters D. Understanding the role of community resilience in addressing the Ebola virus disease epidemic in Liberia: a qualitative study (community resilience in Liberia). Glob Health Action. 2019; 12(1): 1662682.

\section{Correspondencia:}

Candy Palomino-Oré

Dirección: Jr. Eloy Espinoza 709, Urb. Palao, San Martin de Porres. Lima, Perú.

Teléfono: 6149200

Correo electrónico: candypalomino21@gmail.com

\section{Recibido: 17 de abril de 2020}

Evaluado: 06 de mayo de 2020 Aprobado: 14 de mayo de 2020

(c) La revista. Publicado por Universidad de San Martín de Porres, Perú. (c) bajo términos de Licencia Creative Commons Atribución 4.0 Internacional. (http://creativecommons.org/licenses/by/4.0/)

\section{ORCID iDs}

Candy Palomino-Oré

(1) https: / / orcid.org/0000-0002-3576-8234 Jeff Huarcaya-Victoria 